\title{
PROPERTIES OF SOLUTIONS OF CERTAIN SECOND ORDER NONLINEAR DIFFERENTIAL EQUATIONS
}

\author{
W. R. UTZ ${ }^{1}$
}

1. Introduction. In this paper we use theorems on the boundedness of solutions of second order nonlinear differential equations as a starting place for a more detailed examination of the behavior of solutions of such equations for large values of the independent variable. The boundedness theorems we cite give sufficient conditions for the existence of constants $A, B>0$ such that if $x=x(t)$ is a solution of the given equation valid for all large $t$, then $x(t)<B$ for all $t>A$ where $A$ and $B$ may depend upon the particular solution $x=x(t)$. Theorems which guarantee $A, B>0$, as above, but in which $B$ is independent of the solution selected, have been used $[2 ; 4]$ to show the existence and uniqueness of periodic solutions.

If $d>0$ and $e>0$ are real constants, then a solution $x=x(t)$, $x(t) \not \equiv 0$, of the linear equation

$$
x^{\prime \prime}+d x^{\prime}+e x=0
$$

is oscillatory or monotonically approaches zero. The theorems secured in this paper may be thought of as generalizations of this remark.

In $\$ 2$ we state the theorems proved in the paper and in $\$ 3$ provide the proofs of the theorems.

2. Statements of the theorems. Throughout the paper we assume that the coefficients in our equations are differentiable. The function $x(t) \equiv 0$ is assumed to be a solution of each equation considered. We shall use the fact that, because of the uniqueness of solutions, no solution not identically zero can be tangent to the $t$-axis. When we describe a solution $x=x(t)$ of an equation as oscillatory, we shall mean that it has positive maxima for arbitrarily large $t$ and has negative minima for arbitrarily large $t$.

In the first two theorems we consider the equation

$$
x^{\prime \prime}+f\left(x, x^{\prime}\right)+g(x)=x^{\prime} 0, \quad x^{\prime}=d x / d t .
$$

Theorem 1. If $f\left(x, x^{\prime}\right) \geqq 0$ for all $\left(x, x^{\prime}\right), x g(x)>0$ for all $x \neq 0$,

Received by the editors September 4, 1956 and, in revised form, April 5, 1957.

1 This research was supported by the United States Air Force through the Air Force Office of Scientific Research of the Air Research and Development Command under Contract No. AF 18(600)-1108. 


$$
\int_{0}^{x} g(x) d x \rightarrow \infty \text { as } x \rightarrow \infty,
$$

and if $x=x(t) \quad(x(t) \neq 0)$ is a solution of (1) valid for all large t, then $x=x(t)$ is bounded and oscillatory as $t \rightarrow \infty$ or $x(t)$ monotonically approaches zero as $t \rightarrow \infty$.

By making further restriction on $f$ and $g$ we are able to conclude that the amplitudes of the oscillations of an oscillatory solution of (1) will decrease monotonically and hence (1) will not, under these conditions, possess a nontrivial periodic solution. That some restrictions are necessary is clear by observing that if $f\left(x, x^{\prime}\right) \equiv 0$ and $g(x)=x$ then (1) is satisfied by $x=\sin t$.

Theorem 2. If $f\left(x, x^{\prime}\right)>0$ except at a discrete set of points, $g(x)$ is an odd function and $x=x(t)$ is an oscillatory solution of (1), then the amplitudes of the oscillations of this solution are monotonically decreasing.

In the remaining theorems we specialize (1) by insisting that the damping coefficient be independent of $x^{\prime}$ and secure theorems similar to Theorem 1 in which we now allow the damping coefficient to take on negative values.

Theorem 3. Let $f^{\prime}(x)$ denote $d f / d x$ and let $x g(x)>0$ for all $x \neq 0$, $g(x) / x \rightarrow \infty$ as $x \rightarrow \infty$ and suppose that there exist constants $b, B>0$ such that for all real $x$,

$$
|f(x)-b g(x)| \leqq B|x|
$$

If $x=x(t), x(t) \not \equiv 0$, is a solution of

$$
x^{\prime \prime}+f^{\prime}(x) x^{\prime}+g(x)=0
$$

valid for all large $t$, then $x=x(t)$ is bounded and oscillatory or $x(t)$ monotonically approaches zero as $t \rightarrow \infty$.

Theorem 4. Suppose there exist positive constants $\mu$ and $\lambda$ such that

where

$$
\lambda+4 \mu^{2} \geqq \mu F(x) \geqq G(x) \geqq \lambda>0, \quad \lambda>\mu^{2},
$$

$$
F(x)=f(x) / x, \quad G(x)=g(x) / x .
$$

If $x=x(t), x(t) \not \equiv 0$, is any solution of (2) valid for all large $t$, then $x=x(t)$ is bounded and oscillatory or $x(t)$ monotonically approaches zero as $t \rightarrow \infty$.

Examples are easily constructed to show that Theorems 1, 3, 4 are independent. 
If we let $f\left(x, x^{\prime}\right)=1, g(x)=x /\left(x^{2}+1\right)$ in (1) we secure an equation to which Theorem 1 applies but to which Theorems 3, 4 do not apply. If we let $f(x)=g(x)=9 x\left(e^{-x^{2}}+x^{2}\right), b=B=1$ in (2) then Theorem 3 applies to the resulting equation but Theorems 1,4 do not. Finally, if in (2) we let $f(x)=3 x\left(e^{-x^{2}}+1\right), g(x)=3 x, \lambda=3, \mu=3 / 2$, then Theorem 4 applies but Theorems 1, 3 do not apply.

If $g(x)=x^{3}$ and $f(x)=3 x\left(e^{-x^{2}}+1\right)$ then (2) becomes an equation to which no one of the Theorems 1, 3, 4 apply. However, the resulting equation is included in the following theorem.

THEOREM 5. If $x f(x)>0$ and $x g(x)>0$ for all $x \neq 0$ and if $g(x) / x \rightarrow \infty$ as $x \rightarrow \infty$, then any solution $x=x(t), x(t) \not \equiv 0$, of (2) which is valid for all large $t$ is oscillatory or monotonically tends to zero as $t \rightarrow \infty$.

3. Proofs of the theorems. The following lemma will be useful in the proofs of the theorems.

Leмma. If $x g(x)>0, x \neq 0$ and if $x=x(t)$ is a solution of (1) valid for all large $t$, then $x=x(t), x(t) \not \equiv 0$, is strictly monotone or oscillatory.

Proof. If $x=x(t), x(t) \not \equiv 0$, is a monotone solution of (1) then it must be strictly monotone for when $x^{\prime}(t)=0, x^{\prime \prime}=-g(x)$ and hence $x^{\prime \prime} \neq 0$ when $x \neq 0$. Thus $x^{\prime}(t)=0, x^{\prime \prime}=0$ occur simultaneously only for the solution $x(t) \equiv 0$.

If $x=x(t)$ is a solution of (1) and is not monotone then $x=x(t)$ has maxima for arbitrarily large values of $t$ and minima for arbitrarily large values of $t$. Hence there are arbitrarily large values of $t$ such that $x^{\prime}(t)=0$ and $x^{\prime \prime}(t) \geqq 0$ and, also, there are arbitrarily large values of $t$ for which $x^{\prime}(t)=0$, and $x^{\prime \prime}(t) \leqq 0$. However, when $x^{\prime}(t)=0$, $x^{\prime \prime}=-g(x)$ and since $x g(x)>0$ for $x \neq 0$ and since the only solution of (1) tangent to the $t$-axis is $x(t) \equiv 0$, we see that if $x(t)$ is not monotone then $x=x(t)$ is oscillatory. This completes the proof of the lemma.

Proof of Theorem 1. Antosiewicz [1] has shown that under the hypotheses of the theorem $|x(t)|$ and $\left|x^{\prime}(t)\right|$ are bounded as $t \rightarrow \infty$. Using this result and our lemma we may hereafter assume that for $t \geqq a, x(t)$ is monotone.

Since $x(t)$ is monotone, $\lim _{t \rightarrow \infty} x(t)$ exists. Suppose that this limit is $c \neq 0$. From (1) we have

$$
x^{\prime}(t)=x^{\prime}(a)-\int_{a}^{t} g(x(t)) d t-\int_{a}^{t} f\left(x(t), x^{\prime}(t)\right) x^{\prime}(t) d t
$$

for all $t \geqq a$.

Since $|x(t)|$ and $\left|x^{\prime}(t)\right|$ are bounded and $x(t)$ is strictly monotone, 


$$
\int_{a}^{t} f\left(x, x^{\prime}\right) x^{\prime} d t=\int_{x(a)}^{x(t)} f\left(x, x^{\prime}\right) d x
$$

is bounded. However, since $\lim _{t \rightarrow \infty} x(t)=c \neq 0$,

$$
\lim _{t \rightarrow \infty} \int_{a}^{t} g(x) d t= \pm \infty .
$$

From (3) we see that this implies that $x^{\prime}(t)$ is unbounded contrary to Antosiewicz's result and the theorem is proved.

Proof of Theorem 2. If $t_{1}<t_{2}<t_{3}$ are such that $x^{\prime}\left(t_{1}\right)=0, x\left(t_{2}\right)=0$, $x^{\prime}\left(t_{3}\right)=0$, then from equation (1) we secure

$$
x^{\prime 2}\left(t_{2}\right)=-\int_{t_{1}}^{t_{2}} f\left(x, x^{\prime}\right) x^{\prime 2} d t-\int_{x\left(t_{1}\right)}^{x\left(t_{2}\right)} g(x) d x
$$

and

$$
-{x^{\prime}}^{2}\left(t_{2}\right)=-\int_{t_{2}}^{t_{3}} f\left(x, x^{\prime}\right){x^{\prime}}^{2} d t-\int_{x\left(t_{2}\right)}^{x\left(t_{3}\right)} g(x) d x
$$

from which it follows that

$$
\int_{x\left(t_{3}\right)}^{x\left(t_{1}\right)} g(x) d x=\int_{t_{1}}^{t_{3}} f\left(x, x^{\prime}\right) x^{\prime} \quad d t>0 .
$$

Since $g(x)$ is odd and $x\left(t_{2}\right)=0$ it follows that $\left|x\left(t_{1}\right)\right|>\left|x\left(t_{3}\right)\right|$ and the theorem is proved.

Proofs of Theorems 3, 4. It is known [3] that under the hypotheses of this theorem any solution $x=x(t)$ is bounded as $t \rightarrow \infty$. Using this result and our lemma it only remains to show that if $x=x(t)$ is monotone, then $x(t) \rightarrow 0$ as $t \rightarrow \infty$.

Suppose, as in the proof of Theorem $1, x(t)$ is monotone and $\lim _{t \rightarrow \infty} x(t)=c \neq 0$. As in the proof of Theorem 1 , we conclude that $x^{\prime}(t)$ is unbounded. If $g(c)$ is positive, then corresponding to $M<0$ there exists $N>0$ such that for $t>N, x^{\prime}(t)<M$. However, since $x(t) \rightarrow c$ when $t \rightarrow \infty$ and since $c$ is finite this is impossible. A parallel argument holds when $g(c)<0$ and the theorem is proved.

Using another known [3] boundedness theorem one can use the proof given for Theorem 3 to prove Theorem 4 .

Proof of Theorem 5. Again we use our lemma to dispose of all cases except $x(t)$ monotone.

Consider the system

$$
x^{\prime}+f(x)=y, \quad y^{\prime}+g(x)=0
$$


equivalent to (2). For the solution under consideration there exists a constant $a$ such that for $t \geqq a, x=x(t)$ is one sign and strictly monotone. Consider the function

$$
Q(t)=2 \int_{a}^{t} g(x(t)) x^{\prime}(t) d t+y^{2} .
$$

Using (4) we see that for all $t \geqq a$,

$$
d Q / d t=-2 g(x(t)) f(x(t))<0 .
$$

If $x(t)>0, x^{\prime}(t) \geqq 0$ for $t \geqq a$ or if $x(t)<0, x^{\prime}(t) \leqq 0$ for $t \geqq a$ then, since $g(x) / x \rightarrow \infty$ as $x \rightarrow \infty$, if $a$ is sufficiently large

$$
0 \leqq y^{2}(t)+x^{2}(t)-x^{2}(a) \leqq Q(t) .
$$

Thus, since $d Q / d t<0$ for $t \geqq a, x(t)$ is bounded. In the cases $x(t)>0$, $x^{\prime}(t) \leqq 0$ for $t \geqq a$ and $x(t)<0, x^{\prime}(t) \geqq 0$ for $t \geqq a$ it is obvious that $x(t)$ is bounded hence we concluded that if $x=x(t)$ is a monotone solution of (2) then $x(t)$, as $t \rightarrow \infty$, is a bounded function.

The proof may be completed by the argument used to prove Theorem 3.

\section{REFERENCES}

1. H. A. Antosiewicz, On non-linear differential equations of the second order with integrable forcing term, J. London Math. Soc. vol. 30 (1955) pp. 64-67.

2. G. E. H. Reuter, On certain nonlinear differential equations with almost periodic solutions, J. London Math. Soc. vol. 26 (1951) pp. 215-221.

3. W. R. Utz, Su una nota di De Castro, Bollettins della Unione Matematica Italiana (3) vol. 11 (1956) pp. 28-30.

4. Taro Yoshizawa, On the convergence of solutions of the non-linear differential equation, Memoirs of the College of Science, University of Kyoto, Series A, vol. 28 (1953) pp. 143-151.

UNIVERSITY OF MISSOURI 